Jordana Yahr, DO, Juan Calle, MD and Jonathan J. Taliercio*, DO

\title{
A renaissance in the treatment of diabetic kidney disease, hypertension in chronic kidney disease, and beyond
}

https://doi.org/10.1515/jom-2021-0150

Received May 16, 2021; accepted July 20, 2021;

published online October 15, 2021

\begin{abstract}
Chronic kidney disease (CKD) affects approximately $15 \%$ of the US population and is associated with significant cardiovascular morbidity and mortality. The two leading causes of end stage kidney disease are hypertension and diabetes mellitus, both of which are modifiable risk factors. The cornerstones of CKD care include early detection, management of associated risk factors, modification of cardiovascular disease risk, slowing progression of disease, and management of complications including anemia, acid base disturbance, and mineral and bone disorders. For the last 20 years, reninangiotensin system inhibitors were the mainstay treatment for proteinuric diabetic and nondiabetic kidney disease. Recently, new therapies such as sodium-glucose linked transporter 2 inhibitors, have emerged as powerful tools in the treatment of CKD with indications in both diabetic and nondiabetic kidney disease. In this article, we define CKD staging, review new hypertension and diabetic guidelines for CKD patients, and discuss major trials for new potential therapies in CKD, particularly diabetic kidney disease. We will provide practical guidance for primary care physicians to diagnose CKD and implement these agents early in the disease course to prevent the progression of disease and reduce the morbidity and mortality of this vulnerable population.
\end{abstract}

*Corresponding author: Jonathan J. Taliercio, DO, Program Director, Nephrology and Hypertension Fellowship, Assistant Professor of Medicine, Department of Nephrology and Hypertension, Cleveland Clinic Lerner College of Medicine of Case Western Reserve University, Glickman Urological and Kidney Institute, 9500 Euclid Avenue, Q7, Cleveland, OH, 44195, USA, E-mail: talierj@ccf.org Jordana Yahr, DO, Department of Internal Medicine, Cleveland Clinic Foundation, Cleveland, $\mathrm{OH}$, USA

Juan Calle, MD, Department of Nephrology and Hypertension, Cleveland Clinic Lerner College of Medicine of Case Western Reserve University, Cleveland, $\mathrm{OH}$, USA
Keywords: blood pressure; chronic kidney disease; end stage kidney disease; proteinuria; SGTL-2 inhibitor.

Chronic kidney disease (CKD) is estimated to affect $14.9 \%$ of the US adult population and is associated with significant cardiovascular morbidity and mortality [1]. Less than $2 \%$ of those with CKD will progress to end stage kidney or renal disease (ESKD or ESRD) [2]. As of 2018, there were 554,038 adults in the United States on dialysis and an additional 229,887 adults living with a functioning kidney transplant [1]. Those on hemodialysis have a median survival of 47 months [1]. Total expenditures for beneficiaries with ESKD increased to $\$ 49.2$ billion in 2018, which accounted for $7.2 \%$ of the overall Medicare fee-for-service budget [1]. The two leading causes of ESKD are diabetes mellitus (39\%) and hypertension (26\%), both of which are modifiable risk factors [1]. The goals of CKD management can be separated into different aims including: early detection of CKD and identifying etiology, slowing the progression of CKD, addressing cardiovascular risk factors, managing medical complications of CKD (anemia, metabolic acidosis, and secondary hyperparathyroidism), and preparing patients for transition to dialysis, transplant, or conservative care. Primary care providers (PCPs) are critical in CKD care through recognizing the disease early, managing risk factor modification through patient education, achieving diabetic and blood pressure (BP) targets, and initiating renoprotective treatments early in the disease course. In this article, we will define CKD staging and discuss, review, and compare the new hypertension guidelines from 2021 Kidney Disease Improving Global Outcomes (KDIGO) and 2017 American College of Cardiology/American Heart Association (ACC/AHA). In addition, this article will highlight new therapies for CKD patients, specifically for the treatment of diabetic kidney disease (DKD).

\section{Methods}

We performed a search of primary and secondary indexed, peer-reviewed literature through May 2021 utilizing 


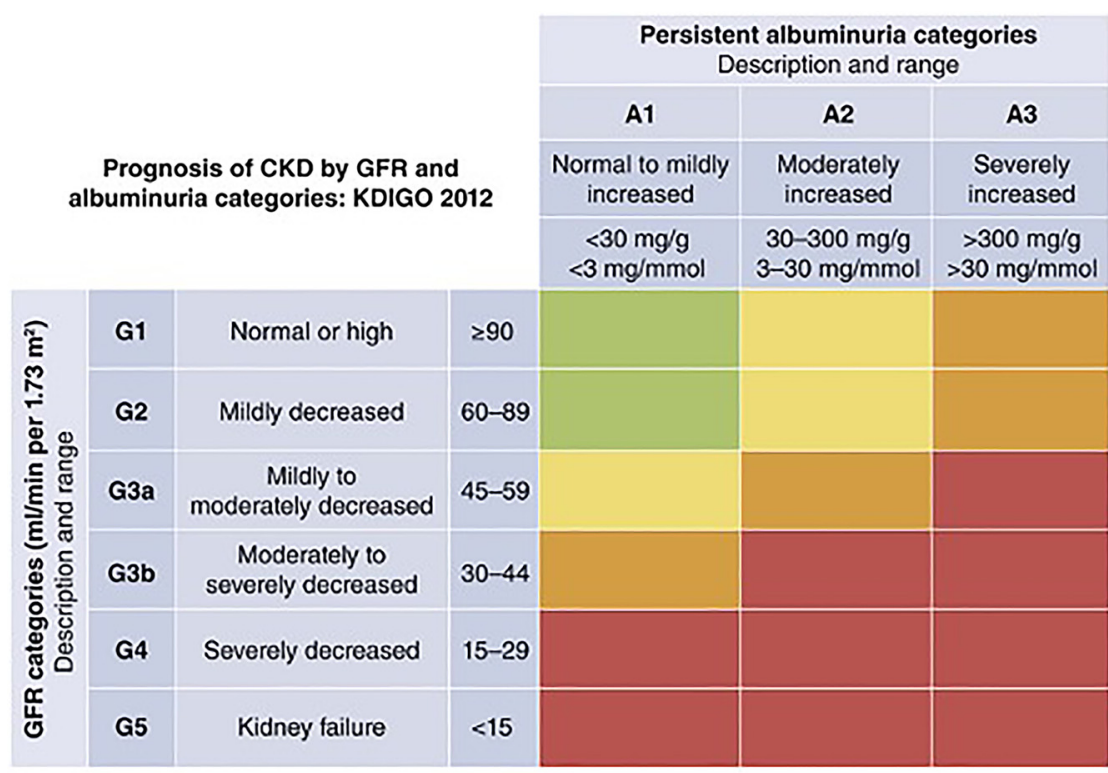

Green, low risk (if no other markers of kidney disease, no CKD); yellow, moderately increased risk; orange, high risk; rod, vory high risk.

Figure 1: Prognosis of CKD by GFR and albuminuria categories. Reproduced with permission from Kidney International [9]. CKD, chronic kidney disease; GFR, glomerular filtration rate.

PubMed and Google Scholar (JT, JY). We utilized a search string to include the words chronic kidney disease, KDIGO, guidelines, American Heart Association, epidemiology, prevalence, risk factors, diabetic kidney disease, proteinuria, albuminuria, treatment, RAS inhibitor, SGLT-2 Inhibitor, GLP-1 receptor agonist, mineralocorticoid receptor antagonist (MRA), and endothelin-A receptor antagonist. The reviewed article types included clinical trials, metaanalyses, systematic reviews, practice guidelines, and pharmaceutical drug information. A total of 50 articles have been included in this review. The authors reviewed all of the results, and disagreements were addressed based on level of expertise (JT).

\section{Discussion}

\section{Defining chronic kidney disease}

The 2012 KDIGO guidelines defines CKD as an estimated glomerular filtration rate (eGFR) of $<60 \mathrm{~mL} / \mathrm{min} / 1.73 \mathrm{~m}^{2}$ for 3 months or an eGFR $\geq 60 \mathrm{~mL} / \mathrm{min} / 1.73 \mathrm{~m}^{2}$ with a structural abnormality for $>3$ months [3]. An abnormality may be defined as albuminuria, abnormalities detected in urine sediment or histology, structural changes seen on imaging, or history of kidney transplant. CKD is classified by the glomerular filtration rate (GFR) category and degree of albuminuria (Figure 1). The 2012 KDIGO heat map differs from the older CKD staging systems by emphasizing the presence and quantity of albuminuria and its impact on CKD progression. It also created a CKD stage 3a (eGFR 45$\left.59 \mathrm{~mL} / \mathrm{min} / 1.73 \mathrm{~m}^{2}\right)$ and $3 \mathrm{~b}\left(30-44 \mathrm{~mL} / \mathrm{min} / 1.73 \mathrm{~m}^{2}\right)$ to help risk-stratify patients. An independent and graded association of reduced eGFR and risk of death, cardiovascular events, and hospitalization has been shown [4]. KDIGO suggests that patients with an eGFR $<30 \mathrm{~mL} / \mathrm{min} / 1.73 \mathrm{~m}^{2}$, urinary albumin-to-creatinine ratio (UACR) $>300 \mathrm{mg} / \mathrm{g}$, or protein-to-creatinine ratio (UPCR) $>500 \mathrm{mg} / \mathrm{g}$ should be referred to a nephrologist. However, we suggest early nephrology intervention because studies have shown that earlier referral to a CKD clinic is associated with a decrease in mortality and stabilization of kidney function $[5,6]$. The KDIGO heat map provides a powerful visual in assessing the risk of progression to ESKD and highlights the need for disease-altering interventions that can be implemented early on to prevent this progression. Although a complete discussion is out of the scope of this article, readers should be aware that most equations for eGFR use race as a variable such as CKD MDRD (Modification of Diet in Renal Disease) equation and the 2009 CKD EPI equation (Epidemiology Collaboration) [7]. The National Kidney Foundation (NKF) and the American Society of Nephrology (ASN) developed a task force in August 2020 on reasessing the inclusion of race in diagnosing kidney disease. The final report was released on September 23, 2021 which recommends using the eGFR 2021 CKD EPI creatinine equation 
that estimates kidney function without a race variable with the goal of providing health equity [8]. Based on these recommendations, many laboratories are starting to implement changes on how they report eGFR. Online calculators for the eGFR 2021 CKD EPI equation may be found by visiting https://www.kidney.org/professionals/kdoqi/ gfr_calculator.

\section{Blood pressure targets in patients with CKD}

Hypertension is both an etiology and complication of CKD $[10,11]$. In a large cross-sectional study of 3,612 patients with CKD, hypertension was seen in up to $85.7 \%$ of participants, and only $46.1 \%$ were controlled below a systolic blood pressure (SBP)/diastolic blood pressure (DBP) $<130 / 80 \mathrm{mmHg}$ [12]. The mechanism of hypertension in CKD patients includes increased salt and volume retention, upregulated reninangiotensin system (RAS), endothelial dysfunction, and increased sympathetic activity [13]. Kidney parenchymal disease is a strong risk factor for resistant hypertension [14]. Resistant hypertension is defined as BP that remains above goal despite the use of three antihypertensive medications at optimal doses, one of which is a diuretic or when goal BP is achieved but requires four medications $[15,16]$. Additionally, in one study of 1,075 adults with CKD, 30.9\% were shown to have masked hypertension (defined as normal office BPs but elevated home pressures) [17]. Elevated BP has been shown to be an independent risk factor for the progression of CKD and the development of ESKD, thus management of hypertension is a crucial component of CKD care [18].

Prior to the 2015 Systolic Blood Pressure Intervention Trial (SPRINT), many guidelines supported a BP goal $<140 / 90 \mathrm{mmHg}$ and a goal $<130 / 80 \mathrm{mmHg}$ for proteinuric kidney disease (defined as urine albumin excretion $\geq 30 \mathrm{mg}$ in $24 \mathrm{~h}$ ) [3, 19]. SPRINT, which enrolled 9,361 non-diabetic patients, was stopped early after a median follow-up of 3.26 years after demonstrating lower all-cause mortality (hazard ratio, [HR] 0.73; 95\% confidence interval [CI], 0.60 to $0.90 ; \mathrm{p}=0.003$ ) and composite outcome (HR 0.75; 95\% CI, 0.64 to $0.89 ; \mathrm{p}<0.001$ ) with more intensive $\mathrm{BP}$ lowering (SBP $<120 \mathrm{mmHg}$ ) vs. standard BP control (SBP $<140 \mathrm{mmHg}$ ) [20]. The primary composite outcome was myocardial infarction (MI), acute coronary syndrome, stroke, heart failure, or death from cardiovascular cause. In a prespecified subgroup analysis of CKD patients, SPRINT Investigators identified that the intensive BP group had a slightly higher rate of decline in eGFR $\left(0.47 \mathrm{vs} .0 .32 \mathrm{~mL} / \mathrm{min} / 1.73 \mathrm{~m}^{2}\right.$ per year; $\mathrm{p}<0.03$ ) and that the overall rate of serious adverse events did not differ between treatment groups. The authors concluded that targeting a SBP $<120 \mathrm{mmHg}$ in patients with
Table 1: Comparison of blood pressure target guidelines.

\begin{tabular}{llll}
\hline Population & $\begin{array}{l}\text { James et al. } \\
\text { (2014 JNC 8) }\end{array}$ & $\begin{array}{l}\text { Whelton et al. } \\
(2017 \text { ACC/AHA) } \\
{[\text { [19] }}\end{array}$ & $\begin{array}{l}\text { Cheung et al. (2021 } \\
\text { KDIGO) [15] }\end{array}$ \\
\hline General & $<140 / 90$ & $<130 / 80$ & No recommendation \\
Diabetes & $<140 / 90$ & $<130 / 80$ & No recommendation \\
CKD & $<140 / 90$ & $<130 / 80$ & $<120^{\mathrm{b}}$ \\
Elderly & $<150 / 90$ & $<130 / 80^{\mathrm{a}}$ & No recommendation \\
& $(\geq 60$ years $)$ & $(\geq 80$ years $)$ & \\
\hline
\end{tabular}

CKD, chronic kidney disease. ${ }^{a}$ Randomized controlled trials have proven beneficial for blood pressure goals $<130 / 80 \mathrm{mmHg}$ in functional elderly patients [20]. ${ }^{\mathrm{b}} 2021 \mathrm{KDIGO}$ guidelines provide recommendations for systolic blood pressure only. 'May be reasonable to have less intensive BP-lowering therapy in patients with very limited life expectancy or symptomatic postural hypotension.

CKD would reduce major cardiovascular events and allcause death without adversely impacting clinical kidney events [21]. As a result, the 2017 ACC/AHA revised their hypertension staging and target guidelines to incorporate these results and suggested targeting $\mathrm{SBP}<130 \mathrm{mmHg}$ and DBP $<80 \mathrm{mmHg}$ for patients with CKD (Level 1 Evidence) [22]. (Table 1)

In March 2021, KDIGO published new hypertension management guidelines for adult patients with CKD. They suggest a more intensive SBP target of $<120 \mathrm{mmHg}$ for adults with CKD with or without diabetes and make no statement of DBP or differentiation between patients with or without albuminuria [15]. These guidelines are clearly influenced from the previously discussed SPRINT trial, but it should be noted that the achieved SBP/DBP of the intensive BP group was $121.4 \mathrm{mmHg} / 68.7 \mathrm{mmHg}$ compared to standard treatment $136.2 \mathrm{mmHg} / 76.3 \mathrm{mmHg}$. These new recommendations are driven by the cardiovascular and mortality benefits and not necessarily kidney protection. For patients with reduced life expectancy or symptomatic postural hypotension, a less intensive BP target should be considered. There were no BP recommendations in the guidelines for the general population, diabetics without CKD, and the functional elderly.

\section{Review of the guidelines: blood pressure treatment in patients with CKD}

Physicians should educate patients on lifestyle modification to achieve BP targets. The cornerstone of all therapy includes restricting sodium to $<2 \mathrm{~g}$ per day, physical activity (moderate intensity, $150 \mathrm{~min}$ per week), weight loss if needed, and limiting alcohol intake (men and women $\leq 2$ and $\leq 1$ drinks per day respectively) [15]. First-line antihypertensive agents should be optimized and should include a 
thiazide diuretic (preferably chlorthalidone), a dihydropyridine calcium channel blocker (preferably amlodipine or long-acting nifedipine), or either an angiotensin converting enzyme inhibitor (ACEi) or an angiotensin receptor blocker (ARB) [22]. Beta-blockers should be first-line therapy only if patients have a compelling indication such as heart disease or heart failure [22]. Other comorbidities, like diabetes status, should be taken into account when choosing a first-line agent. Chlorthalidone (12.5-25 mg/day) has re-emerged as the diuretic of choice due to a longer half-life and effectiveness in lower eGFR $\left(\sim 25 \mathrm{~mL} / \mathrm{min} / 1.73 \mathrm{~m}^{2}\right)$ compared to hydrochlorothiazide [22]. If eGFR $\leq 30 \mathrm{~mL} / \mathrm{min} / 1.73 \mathrm{~m}^{2}$ and hypertension is not controlled, the diuretic should be switched to a loop [22]. Mineralocorticoid receptor antagonists (MRAs) are recommended as fourth-line agents in resistant hypertension [15]. Providers should be aware of the increased risk of hyperkalemia associated with this class of medication [15]. Patients with an average SBP/DBP >20/ $10 \mathrm{mmHg}$ above their target should be initiated on two firstline agents [22].

\section{RAS inhibition in CKD}

The renoprotective and cardioprotective benefits of RAS inhibitors, including ACEis and ARBs, have been well demonstrated in multiple subgroups of CKD including those with and without diabetes [23]. It has been over 25 years since the Collaborative Study Group (The Captopril Trial) demonstrated the renoprotective benefits of RAS inhibition in proteinuric type 1 diabetes mellitus [24]. In 2001, the Irbesartan Diabetic Nephropathy Trial (IDNT) studied 1,715 type 2 diabetes mellitus (T2DM) subjects with proteinuria $\geq 900 \mathrm{mg} /$ day and demonstrated a $20 \%$ risk reduction in composite endpoint of doubling of serum creatinine, ESKD, or death from any cause in the irbesartan treatment group compared to placebo $(95 \% \mathrm{CI}, 0.66-0.97$; $\mathrm{p}=0.02$ ), and $23 \%$ risk reduction compared to amlodipine groups (95\% CI, 0.63-0.93; $\mathrm{p}=0.006$ ) [25]. The Reduction of Endpoints in NIDDM with the Angiotensin II Antagonist Losartan (RENAAL) trial studied 1,513 subjects with T2DM with a UACR $\geq 300 \mathrm{mg} / \mathrm{g}$ and showed a $16 \%$ risk reduction in the primary composite endpoint of doubling of serum creatinine, ESKD, or death from any cause in the losartan treatment group compared to the placebo group (43.5 vs. 47.1\%, $\mathrm{p}=0.02$ ) [26]. The renoprotective effects of ACEis and ARBs result from the vasodilatory properties that they have on the renal efferent arteriole, which lowers intraglomerular hypertension and reduces GFR [27]. An acute fall in eGFR has been correlated with improved kidney outcomes [27]. The ACC/AHA guidelines recommend treatment with ACEi or ARB in patients with CKD $\geq$ stage 3 regardless of albuminuria AND in all patients with albuminuria $\geq 300 \mathrm{mg} /$ day or UACR $\geq 300 \mathrm{mg} / \mathrm{g}$ [22].

It is important for physicians to be aware of risks of ACEis and ARBs including acute kidney injury (AKI) and hyperkalemia. A decrease in eGFR may be seen upon initiating an ACEi or ARB due to a reduction in intraglomerular pressures [27]. Current KDIGO 2021 guidelines recommend that a rise in serum creatinine up to $30 \%$ is acceptable, as long as it is not associated with other complications such as hyperkalemia. A drop in eGFR $>30 \%$ may suggest bilateral renal artery stenosis or patients with volume depletion [15]. Renal function and electrolytes should be checked within 2-4 weeks of initiation or increasing the dose of RAS inhibitors. Patients should continue maximal ACEi or ARB therapy unless they experience hyperkalemia not amendable to medical therapy. Medical therapy includes reducing potassium intake to 2,000-3,000 mg per day (50-75 meq per day), discontinuation of potassium-sparing diuretics, and initiating oral potassium-binding agents (patiromer or sodium zirconium cyclosilicate) if needed. The benefits of ACEi or ARB therapy should be prioritized, and the previously mentioned measures should be considered prior to dose reduction or discontinuation of therapy. ACEi and ARB are not recommended to be utilized concomitantly because of an increased risk of acute kidney injury and hyperkalemia [15]. In certain patients, it may be necessary to discontinue ACEis or ARBs for refractory hyperkalemia, particularly in the population transitioning to kidney replacement therapy (KRT).

\section{Major advances in diabetic kidney disease}

\section{SGLT-2 inhibitors}

Since the landmark trials of RAS inhibition, there has been a paucity of treatment options for patients with CKD. The sodium-glucose cotransporter-2 (SGLT-2) inhibitors have opened the door to a new era as add-on therapy proven to slow the progression of CKD. SGLT-2 inhibitors are rapidly changing practice patterns. The 2020 KDIGO Practice Guidelines for Diabetic Management in CKD goes beyond the 2020 American Diabetic Association and American Association of Clinical Endocrinology recommending SGLT-2 inhibitors $A N D$ metformin as first-line drugs for DKD [9]. The recommendation to add SGLT-2 inhibitors in combination with metformin is primarily for the beneficial 
effects of slowing the progression of kidney and cardiovascular disease. The KDIGO guideline specifically references data from Dapagliflozin and Prevention of Adverse Outcomes in Chronic Kidney Disease (DAPA-CKD), Dapagliflozin and Prevention of Adverse Outcomes in Heart Failure (DAPA-HF), and Empagliflozin Outcome Trial in Patients with Chronic Heart Failure and a Reduced Ejection Fraction (EMPEROR-Reduced) trials that showed benefits in cardiovascular outcomes in both those with and without diabetes, suggesting that the mechanism of benefit was not purely due to glucose control [28-30]. The recommendation goes as far as to recommend decreasing the dose of other antihyperglycemic agents for patients who have met their glycemic goal if they are on agents that put them at risk for hypoglycemia in order to add on an SGLT-2 inhibitor.

The Empagliflozin and Progression of Kidney Disease in Type 2 Diabetes, a prespecified secondary analysis of the Empagliflozin, Cardiovascular Outcomes, and Mortality in T2DM (EMPA-REG Outcomes) trial, showed a reduction in kidney composite outcomes (doubling of serum creatinine, initiation of KRT, death from kidney disease) in those with T2DM (HR 0.54; 95\% CI, 0.4-0.75; p<0.001) [31, 32]. The first major randomized placebo-controlled multicenter study to evaluate primary kidney composite outcomes (doubling of baseline serum creatinine, development of ESKD, or death from a renal or cardiovascular cause) with SGLT-2 inhibitors vs. placebo was the Canagliflozin and Renal Events in Diabetes with Established Nephropathy Clinical Evaluation (CREDENCE) trial [33]. Approximately 4,400 T2DM patients with eGFR $30-90 \mathrm{~mL} / \mathrm{min} / 1.73 \mathrm{~m}^{2}$ and albuminuria (UACR $>300-5,000 \mathrm{mg} / \mathrm{g}$ ) treated with optimal doses of RAS blockade were randomized to canagliflozin $100 \mathrm{mg}$ daily or placebo. After a planned interim analysis, the trial was stopped early after a median follow-up of 2.62 years due to the overwhelming beneficial kidney protection. The canagliflozin group experienced a 30\% lower risk of kidney events (HR 0.70; 95\% CI, 0.59 to 0.82; $\mathrm{p}=0.00001$ ) compared to placebo. This astonishing outcome was seen in patients who were already taking maximally tolerated ACEis or ARBs. Comparing the previously discussed IDNT and RENAAL trials, the relative risk of doubling of serum creatinine was $40 \%$ lower in the CREDENCE trial (HR 0.6; 95\% CI, 0.48 to $0.76 ; \mathrm{p}<0.001)$, compared to the other two studies respectively $29 \%$ (HR $0.71 ; 95 \% \mathrm{CI}, 0.54$ to $0.92 ; \mathrm{p}=0.009$ ) and 25\% (HR 0.75; 95\% CI, 8 to 39; $\mathrm{p}=0.006$ ). Postulated mechanisms for the renoprotective effects of SGLT- 2 inhibitors include a decrease in intraglomerular pressure via indirect vasoconstriction of afferent arteriole via tubuloglomerular feedback, anti-inflammatory, and antioxidant by inducing a starvation state via ketonemia [34, 35].
The CREDENCE trial proved the benefit of SGLT- 2 inhibitors for DKD, and the DAPA-CKD trial expanded the indication to proteinuric CKD patients without DKD [30]. The DAPA-CKD trial enrolled 4,304 CKD subjects on maximal RAS blockade with lower eGFRs $\left(25-75 \mathrm{~mL} / \mathrm{min} / 1.73 \mathrm{~m}^{2}\right)$, lesser degree of proteinuria, UACR $(\geq 200-5,000 \mathrm{mg} / \mathrm{g})$, with or without T2DM. Patients received dapagliflozin (10 mg daily) or placebo, and the primary kidney outcome was sustained decline in eGFR of at least $50 \%$, ESKD, or death from kidney or cardiovascular disease [30]. The study was stopped early after 2.4 years because there was a $39 \%$ reduction in the primary composite outcome in the dapagliflozin group (HR 0.61; 95\% CI, 0.51 to $0.72 ; \mathrm{p}<0.001$ ). The results were similar in T2DM and non-diabetics, which comprised 67 and 33\% of the subjects, respectively. A prespecified analysis of a subgroup of DAPA-CKD patients who had concomitant IgA nephropathy ( $\mathrm{n}=270)$, had a $71 \%$ reduction in primary outcomes (HR 0.29; 95\% CI, 0.12 to 0.73; $\mathrm{p}<0.005)$ suggesting that SGLT-2 inhibitors could be utilized in non-diabetic proteinuric kidney disease [36]. These results led to a new FDA-prescribing indication for dapagliflozin on April 30, 2021, for use in the management of CKD with or without T2DM. The Study of Heart and Kidney Protection with Empagliflozin (EMPA-KIDNEY) trial is evaluating 6,000 CKD patients (eGFR $\geq 20-89 \mathrm{~mL} / \mathrm{min} / 1.73 \mathrm{~m}^{2}$ ) with or without T2DM and is planned to be completed by October 2022 [37].

Providers should be aware that SGLT- 2 inhibitors may cause a $5-8 \mathrm{ml} / \mathrm{min} / 1.73 \mathrm{~m}^{2}$ drop in eGFR in the first 2 weeks of therapy due to the hemodynamic effects similar to those seen in ACEis and ARBs [38]. Prescribers should discuss the risk and benefits of urinary tract infections, diabetic ketoacidosis, and the propensity for volume depletion on "sick days" or in patients who are on concomitant diuretic therapy [39].

\section{GLP-1 receptor agonists}

In addition to SGLT-2 inhibitors, other antihyperglycemic agents have shown positive data in the treatment of DKD. Glucagon like peptide-1 (GLP-1) receptor agonists are antihyperglycemics that have been shown to reduce the risk of cardiovascular events in patients with T2DM [40]. GLP-1 receptor agonists work by mimicking the body's natural response to consuming calories, including insulin secretion, appetite suppression, slowing gastric emptying, and utilization of insulin in peripheral tissues [41]. The Liraglutide Effect and Action in Diabetes: Evaluation of Cardiovascular Outcome Results (LEADER) trial enrolled 9,340 patients with T2DM and evaluated a secondary 


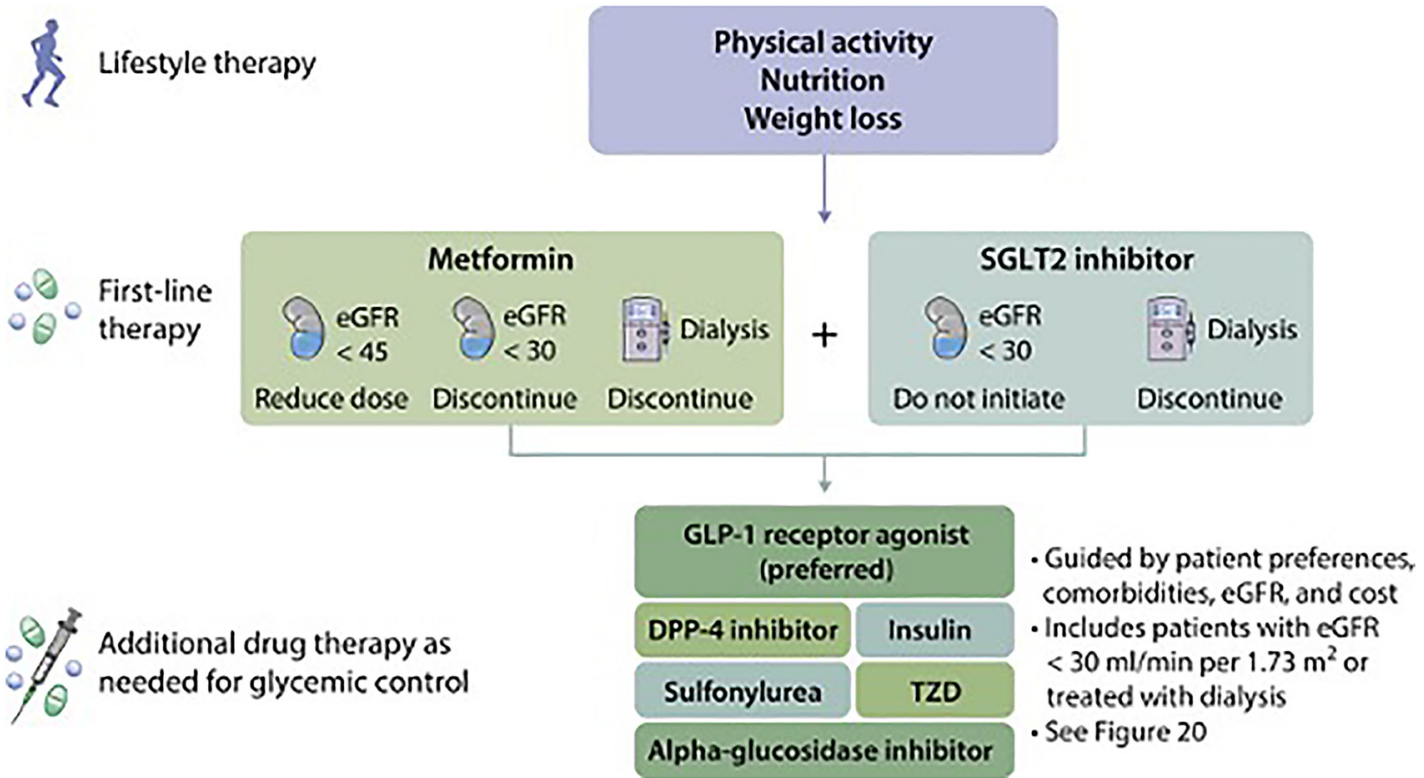

Figure 2: Treatment algorithm for selecting antihyperglycemic drugs for patients with T2D and CKD. Reproduced with permission from Kidney International [9]. Kidney icon indicates eGFR (expressed in $\mathrm{mL} / \mathrm{min}$ per $1.73 \mathrm{~m}^{2}$ ); dialysis machine icon indicates dialysis. CKD, chronic kidney disease; DPP-4, dipeptidyl peptidase-4; eGFR, estimated glomerular filtration rate; GLP-1, glucagon-like peptide-1 (GLP-1); SGLT-2, sodiumglucose cotransporter-2; T2D, type 2 diabetes; TZD, thiazolidinedione.

microvascular, retinal, and kidney composite outcome (defined as new-onset UACR > $300 \mathrm{mg} / \mathrm{g}$, doubling of serum creatinine, and eGFR $\leq 45 \mathrm{~mL} / \mathrm{min} / 1.73 \mathrm{~m}^{2}$, ESKD, or death from renal cause) and retinopathy (defined by the need for retinal photocoagulation or treatment with intravitreal agents, vitreous hemorrhage, or the onset of diabetes-related blindness). The rate of nephropathy events was reduced in the liraglutide group vs. placebo (1.5 vs. 1.9 events per 100 patient-years of observation) (HR 0.78 ; $95 \%$ CI, 0.67 to $0.92, p=0.003$ ) [42]. Renoprotective data was also seen in the Semaglutide and Cardiovascular Outcomes in Patients with Type 2 Diabetes (SUSTAIN-6) trial, which enrolled 3,297 T2DM patients. The semaglutide group vs. placebo had a reduction in nephropathy (HR 0.64; 95\% CI, 0.46-0.88; $p=0.005$ ), but the outcome was primarily driven by the reduction in new-onset UACR $>300 \mathrm{mg} / \mathrm{g}$ [43]. New or worsening nephropathy was defined as new-onset UACR $>300 \mathrm{mg} / \mathrm{g}$, doubling of serum creatinine, and eGFR $\leq 45 \mathrm{~mL} / \mathrm{min} / 1.73 \mathrm{~m}^{2}$, ESKD, or death from a renal cause. In a 2019 meta-analysis, GLP-1 receptor agonists were shown to reduce a primary composite outcome (doubling of serum creatinine, $\geq 40 \%$ decline in eGFR, ESKD, or kidney-related death) by $17 \%$ (HR 0.83; 95\% CI, 0.78 to 0.89 ; $\mathrm{p}<0.001$ ) [44].

Furthermore, the Dulaglutide and Cardiovascular Outcomes in Type 2 Diabetes (REWIND) trial showed a decrease in kidney outcomes (defined as development of a UACR $>33.9 \mathrm{mg} / \mathrm{mmol}$, a sustained $\geq 30 \%$ decline in eGFR, or KRT) in the dulaglutide group vs. placebo (HR 0.85; 95\% CI, 0.77-0.93; $p=0.004$ ) [45]. While SGLT-2 inhibitors and metformin are recommended as first-line therapy for T2DM with CKD, given this data, the 2020 KDIGO Diabetes guidelines recommend GLP-1 receptor agonists as the preferred additional agent for patients with T2DM and CKD whose hemoglobin A1c goals are not met with metformin and an SGLT-2 inhibitor [9]. (Figure 2) Providers should counsel patients on the side effects of GLP-1 receptor agonists, including gastrointestinal distress, which is the most common. Pancreatitis has been associated with GLP-1 agonists and is included in the package insert; however, the LEADER trial demonstrated no association in the liraglutide group compared to placebo [42].

\section{Mineralocorticoid receptor antagonists (MRAs)}

Adding MRAs (spironolactone and eplerenone) to ACEi or ARBs has been shown to reduce albuminuria by approximately $25-30 \%$, but with an increased risk of hyperkalemia (2.6-fold increase) and without much efficacy in reducing CKD progression [46, 47]. Finerenone is a nonsteroidal MRA that has a greater receptor selectivity and affinity than the former two aforementioned MRAs. The effect of the Finerenone in Reducing Kidney Failure and Disease Progression in Diabetic Kidney Disease (FIDELIO-DKD) trial 
enrolled 5,734 patients with T2DM and CKD with UACR of $300-5,000 \mathrm{mg} / \mathrm{g}$ and an eGFR $25-74 \mathrm{~mL} / \mathrm{min} / 1.73 \mathrm{~m}^{2}$ on maximally tolerated RAS inhibition and randomized them to receive finerenone $10 \mathrm{mg}$ or $20 \mathrm{mg}$ daily or placebo [48]. The primary composite endpoint included ESKD, sustained decrease in eGFR $\geq 40 \%$, or death from a kidney cause. The secondary composite outcome was death from a cardiovascular cause, nonfatal MI, nonfatal stroke, or heart failure hospitalization. After 2.6 years of median follow-up, the finerenone group had an 18\% lower incidence of the primary kidney outcome (HR 0.82; 95\% CI, 0.73 to 0.93 ; $\mathrm{p}=0.001$ ) and a lower secondary composite outcome (HR 0.86 ; $95 \% \mathrm{CI}, 0.75$ to $0.99 ; \mathrm{p}=0.03$ ). Incidences of potassium $>5.5 \mathrm{mmol} / \mathrm{L}$ ( $21.7 \mathrm{vs.} 9.8 \%$ ) and $>6.0 \mathrm{mmol} / \mathrm{L}$ ( 4.5 vs. $1.4 \%$ ) were higher in the finerenone group compared to placebo. This led to a higher discontinuation rate in the finerenone group vs. placebo ( 2.3 vs. $0.9 \%$ ). It should be mentioned that only $4.6 \%$ of all participants were treated with an SGLT-2 inhibitor and 6.9\% with a GLP-1 receptor agonist [48]. Therefore, it is unknown whether the beneficial effects of finerenone may be blunted in combination with the former therapies. On July 9, 2021, finerenone was approved by the FDA for use in adults with CKD and T2DM [49].

\section{Endothelin a receptor antagonist}

Endothelin A is a vasoactive peptide that constricts blood vessels that result in systemic and pulmonary hypertension. Endothelin A receptor antagonists are an FDA-approved treatment for pulmonary arterial hypertension and have been studied in resistant hypertension [50]. Endothelin A is a mediator for kidney injury via inflammation, endothelial injury, podocyte disruption, and fibrosis. Endothelin A is a profound vasoconstrictor of the afferent and efferent arterioles, which leads to decreases in GFR [51]. Endothelin A receptor antagonists have been shown to reduce BP and albuminuria in patients with T2DM, but they have been associated with fluid overload and heart failure exacerbation [52]. The Study of Diabetic Retinopathy with Atrasentan (SONAR) trial studied T2DM patients with an eGFR of $25-75 \mathrm{~mL} / \mathrm{min} / 1.73 \mathrm{~m}^{2}$, UACR of $300-5,000 \mathrm{mg} / \mathrm{g}$, on maximally tolerated RAS blockade for a minimum of 4 weeks [53]. All patients were initially treated with atrasentan $0.75 \mathrm{mg}$ daily. Only those patients who responded with $\geq 30 \%$ reduction in UACR and showed "no substantial fluid retention" were then randomized to treatment vs. control. The atrasentan treatment group showed a 35\% reduction (HR 0.65; 95\% CI, 0.49-0.88; $\mathrm{p}=0.0047$ ) in composite kidney outcomes (doubling of serum creatinine, eGFR $<15$, ESKD, kidney transplant, or death from kidney cause), a 52\% reduction in UACR, and a lowering of SBP of $1.6 \mathrm{mmHg}$. There were no differences between the two groups for serious adverse events, but patients taking atrasentan had a higher rate of hypervolemia ( 36.6 vs. $32.3 \%, \mathrm{p}=0.022$ ) and anemia (18.5 vs. $10.3 \%, p=0.0001)$. The trial stopped early after 2.2 years because the primary outcome was lower than expected. Clinical application of this adaptive study design may be more challenging because only study participants who had $a \geq 30 \%$ reduction in albuminuria and no signs of volume retention were studied. Therefore, the actual adverse events due to treatment may be much higher, and the risk of this class of medication may outweigh the benefits.

\section{Conclusions}

All osteopathic physicians who provide primary care have a critical role in the early diagnosis and management of CKD. Guidelines recommend referral to a nephrologist with an eGFR $<30 \mathrm{~mL} / \mathrm{min} / 1.73 \mathrm{~m}^{2}$, UACR > $300 \mathrm{mg} / \mathrm{g}$, or UPCR $>500 \mathrm{mg} / \mathrm{g}$. We advocate for earlier referral with an eGFR $\leq 45 \mathrm{~mL} / \mathrm{min} / 1.73 \mathrm{~m}^{2}$ or if the etiology of CKD is unknown. In addition to lifestyle modification, strict BP control and diabetic control are the cornerstones of therapy. For the last 25 years, the medical community only had ACEi and ARBs to slow disease progression. SGLT-2 inhibitors have provided a renaissance in the treatment of proteinuric CKD. GLP-1 receptor agonists, MRAs, and endothelin A receptor antagonists may provide physicians with a potential armamentarium of targeted kidney disease therapies. PCPs should be the initial prescribers of these therapies early in the course of the disease to maximize their benefits and to reduce the prevalence and progression of CKD. The information presented in this narrative review is based on a review of the literature and clinical expertise, but further research in systematic reviews, meta-analyses, and ongoing randomized controlled trials are indicated.

Research funding: None reported.

Author contributions: All authors provided substantial contributions to conception and design, acquisition of data, or analysis and interpretation of data; all authors drafted the article or revised it critically for important intellectual content; all authors gave final approval of the version of the article to be published; and all authors agree to be accountable for all aspects of the work in ensuring that questions related to the accuracy or integrity of any part of the work are appropriately investigated and resolved. 
Competing interests: Jonathan Taliercio DO, is an EMPA KIDNEY and SONAR Co-investigator.

\section{References}

1. United States Renal Data System. USRDS annual data report: epidemiology of kidney disease in the United States. Bethesda, MD: National Institutes of Health, National Institute of Diabetes and Digestive and Kidney Diseases; 2020.

2. Keith DS, Nichols GA, Gullion CM, Brown JB, Smith DH. Longitudinal follow-up and outcomes among a population with chronic kidney disease in a large managed care organization. Arch Intern Med 2004;164:659-63.

3. Kidney Disease: Improving Global Outcomes (KDIGO) CKD Work Group. KDIGO 2012 clinical practice guideline for the evaluation and management of chronic kidney disease. Kidney Int Suppl 2013;3:1-150.

4. Go AS, Chertow GM, Fan D, McCulloch CE, Hsu C. Chronic kidney disease and the risks of death, cardiovascular events, and hospitalization. N Engl J Med 2004;351:1296-305.

5. Tseng CL. Survival benefit of nephrologic care in patients with diabetes mellitus and chronic kidney disease. Arch Intern Med 2008;168:55.

6. Serrano A, Huang J, Ghossein C, Nishi L, Gangavathi A, Madhan V, et al. Stabilization of glomerular filtration rate in advanced chronic kidney disease: a two-year follow-up of a cohort of chronic kidney disease patients stages 4 and 5. Adv Chron Kidney Dis 2007;14:105-12.

7. Inker LA, Eneanya ND, Coresh J, Tighiouart H, Wang D, Sang Y, et al. New creatinine- and cystatin C-based equations to estimate GFR without race. N Engl J Med 2021. https://doi.org/10.1056/ NEJMoa2102953.

8. National Kidney Foundation. NKF and ASN release new way to diagnose kidney diseases; 2021. Available from: https://www. kidney.org/news/nkf-and-asn-release-new-way-to-diagnosekidney-diseases [Accessed 10 Oct 2021].

9. de Boer IH, Caramori ML, Chan JCN, Heerspink HJL, Hurst C, Khunti K, et al. Executive summary of the 2020 KDIGO Diabetes Management in CKD Guideline: evidence-based advances in monitoring and treatment. Kidney Int 2020;98: 839-48.

10. Bidani AK, Griffin KA. Pathophysiology of hypertensive renal damage: implications for therapy. Hypertension 2004;44: 595-601.

11. Brantsma AH, Bakker SJL, de Zeeuw D, de Jong PE, Gansevoort RT. Urinary albumin excretion as a predictor of the development of hypertension in the general population. J Am Soc Nephrol 2006; 17:331-5.

12. Muntner P, Anderson A, Charleston J, Chen Z, Ford V, Makos G, et al. Hypertension awareness, treatment, and control in adults with CKD. J Am Phys Ther Assoc 2011;55:441-51.

13. Huan Y, Cohen DL, Townsend RR. Pathophysiology of hypertension in chronic kidney disease. In: Kimmel PL, Rosenberg ME, editors. Chronic renal disease. Elsevier; 2015: 163-9 pp.

14. Calhoun DA, Jones D, Textor S, Goff DC, Murphy TP, Toto RD, et al. Resistant hypertension: diagnosis, evaluation, and treatment: a scientific statement from the American heart association professional education committee of the council for high blood pressure research. Circulation 2008;117:e510-26.

15. Cheung AK, Chang TI, Cushman WC, Furth SL, Hou F, Ix JH, et al. KDIGO 2021 clinical practice guideline for the management of blood pressure in chronic kidney disease. Kidney Int 2021;99:S187.

16. Judd E, Calhoun D. Apparent and true resistant hypertension: definition, prevalence and outcomes. J Hum Hypertens 2014;28: 463-8.

17. limuro S, Imai E, Watanabe T, Nitta K, Akizawa T, Matsuo S, et al. Clinical correlates of ambulatory BP monitoring among patients with CKD. Clin J Am Soc Nephrol 2013;8:721-30.

18. Tozawa M, Iseki K, Iseki C, Kinjo K, Ikemiya Y, Takishita S. Blood pressure predicts risk of developing end-stage renal disease in men and women. Hypertension 2003;41:1341-5.

19. James PA, Oparil S, Carter BL, Cushman WC, DennisonHimmelfarb C, Handler J, et al. Evidence-based guideline for the management of high blood pressure in adults: report from the panel members appointed to the eighth joint national committee (JNC 8). J Am Med Assoc 2014;311:507.

20. SPRINT Research Group. A randomized trial of intensive versus standard blood-pressure control. N Engl J Med 2015;373: 2103-16.

21. Cheung AK, Rahman M, Reboussin DM, Craven TE, Greene T, Kimmel PL, et al. Effects of intensive BP control in CKD. J Am Soc Nephrol 2017;28:2812-23.

22. Whelton PK, Carey RM, Aronow WS, Casey DE, Collins KJ, Dennison-Himmelfarb C, et al. ACC/AHA/AAPA/ABC/ACPM/AGS/ APhA/ASH/ASPC/NMA/PCNA guideline for the prevention, detection, evaluation, and management of high blood pressure in adults. J Am Coll Cardiol 2018;71:e127-248.

23. Jafar TH, Stark PC, Schmid CH, Janda M, Maschio G, de Jong PE, et al. Progression of chronic kidney disease: the role of blood pressure control, proteinuria, and angiotensin-converting enzyme inhibition: a patient-level meta-analysis. Ann Intern Med 2003;139:244.

24. Lewis EJ, Hunsicker LG, Bain RP, Rohde RD. The effect of angiotensin-converting-enzyme inhibition on diabetic nephropathy. N Engl J Med. 1993;329:1456-62.

25. Lewis EJ, Hunsicker LG, Clarke WR, Berl T, Pohl MA, Lewis JB, et al. Renoprotective effect of the angiotensin-receptor antagonist irbesartan in patients with nephropathy due to type 2 diabetes. $\mathrm{N}$ Engl J Med 2001;345:851-60.

26. Brenner BM, Cooper ME, de Zeeuw D, Keane WF, Mitch WE, Parving $\mathrm{HH}$, et al. Effects of losartan on renal and cardiovascular outcomes in patients with type 2 diabetes and nephropathy. $\mathrm{N}$ Engl J Med 2001;345:861-9.

27. Holtkamp FA, De Zeeuw D, Thomas MC, Cooper ME, de Graeff PA, Hillege HJL, et al. An acute fall in estimated glomerular filtration rate during treatment with losartan predicts a slower decrease in long-term renal function. Kidney Int 2011;80:282-7.

28. McMurray JJV, Solomon SD, Inzucchi SE, Køber L, Kosiborod MN, Martinez FA, et al. Dapagliflozin in patients with heart failure and reduced ejection fraction. N Engl J Med 2019;381:1995-2008.

29. Packer M, Anker SD, Butler J, Filippatos G, Pocock SJ, Carson P, et al. Cardiovascular and renal outcomes with empagliflozin in heart failure. N Engl J Med 2020;383:1413-24.

30. Heerspink HJL, Stefánsson BV, Correa-Rotter R, Chertow GM, Greene T, Hou FF, et al. Dapagliflozin in patients with chronic kidney disease. N Engl J Med 2020;383:1436-46. 
31. Zinman B, Wanner C, Lachin JM, Fitchett D, Bluhmki E, Hantel S, et al. Empagliflozin, cardiovascular outcomes, and mortality in type 2 diabetes. N Engl J Med 2015;373:2117-28.

32. Wanner C, Inzucchi SE, Lachin JM, Fitchett D, von Eynatten M, Mattheus M, et al. Empagliflozin and progression of kidney disease in type 2 diabetes. N Engl J Med 2016;375:323-34.

33. Perkovic V, Jardine MJ, Neal B, Bompoint S, Heerspink HJL, Charytan DM, et al. Canagliflozin and renal outcomes in type 2 diabetes and nephropathy. N Engl J Med 2019;380:2295-306.

34. van Bommel EJM, Muskiet MHA, Tonneijck L, Kramer MHH, Nieuwdorp M, van Raalte DH. SGLT2 inhibition in the diabetic kidney-from mechanisms to clinical outcome. Clin J Am Soc Nephrol 2017;12:700-10.

35. Packer M. SGLT2 inhibitors produce cardiorenal benefits by promoting adaptive cellular reprogramming to induce a state of fasting mimicry: a paradigm shift in understanding their mechanism of action. Diabetes Care 2020;43:508-11.

36. Wheeler DC, Toto RD, Stefansson BV, Jongs N, Chertow G, Greene $T$, et al. A pre-specified analysis of the DAPA-CKD trial indicates effects of dapagliflozin on major adverse kidney events in patients with IgA nephropathy. Kidney Int 2021;100:215-24.

37. About us-EMPA-kidney. Available from: https://www. empakidney.org/about [Accessed 2 July 2021].

38. Nespoux J, Vallon V. SGLT2 inhibition and kidney protection. Clin Sci Lond Engl 2018;132:1329-39.

39. Taliercio JJ, Thomas G, Nakhoul GN, Vachharajani TJ, Mehdi A. SGLT-2 inhibitors: a new era in managing diabetic kidney disease starts now. Cleve Clin J Med 2020;88:59-63.

40. Boyle JG, Livingstone R, Petrie JR. Cardiovascular benefits of GLP-1 agonists in type 2 diabetes: a comparative review. Clin Sci 2018;132:1699-709.

41. Cornell S. A review of GLP-1 receptor agonists in type 2 diabetes: a focus on the mechanism of action of once-weekly agents. J Clin Pharm Ther 2020;45:17-27.

42. Marso SP, Daniels GH, Brown-Frandsen K, Kristensen P, Mann JFE, Nauck MA, et al. Liraglutide and cardiovascular outcomes in type 2 diabetes. N Engl J Med 2016;375:311-22.

43. Marso SP, Bain SC, Consoli A, Eliaschewitz FG, Jodar E, Leiter LA, et al. Semaglutide and cardiovascular outcomes in patients with type 2 diabetes. N Engl J Med 2016;375:1834-44.

44. Kristensen SL, Rørth R, Jhund PS, Docherty KF, Sattar N, Preiss D, et al. Cardiovascular, mortality, and kidney outcomes with GLP-1 receptor agonists in patients with type 2 diabetes: a systematic review and meta-analysis of cardiovascular outcome trials. Lancet Diabetes Endocrinol 2019;7:776-85.

45. Gerstein HC, Colhoun HM, Dagenais GR, Diaz R, Lakshmanan M, Pais $P$, et al. Dulaglutide and cardiovascular outcomes in type 2 diabetes (REWIND): a double-blind, randomised placebocontrolled trial. Lancet 2019;394:121-30.

46. Navaneethan SD, Nigwekar SU, Sehgal AR, Strippoli GFM. Aldosterone antagonists for preventing the progression of chronic kidney disease: a systematic review and meta-analysis. Clin J Am Soc Nephrol 2009;4:542-51.

47. Alexandrou ME, Papagianni A, Tsapas A, Loutradis C, Boutou A, Piperidou A, et al. Effects of mineralocorticoid receptor antagonists in proteinuric kidney disease: a systematic review and meta-analysis of randomized controlled trials. J Hypertens 2019;37:2307-24.

48. Bakris GL, Agarwal R, Anker SD, Pitt B, Ruilope LM, Rossing P, et al. Effect of finerenone on chronic kidney disease outcomes in type 2 diabetes. N Engl J Med 2020;383:2219-29.

49. US Food and Drug Administration. FDA approves drug to reduce risk of serious kidney and heart complications in adults with chronic kidney disease associated with type 2 diabetes. Available from: https://www.fda.gov/drugs/drug-safety-andavailability/fda-approves-drug-reduce-risk-serious-kidneyand-heart-complications-adults-chronic-kidney-disease [Accessed 30 July 2021].

50. Weber MA, Black H, Bakris G, Krum H, Linas S, Weiss R, et al. A selective endothelin-receptor antagonist to reduce blood pressure in patients with treatment-resistant hypertension: a randomised, double-blind, placebo-controlled trial. Lancet 2009; 374:1423-31.

51. Kohan DE, Barton M. Endothelin and endothelin antagonists in chronic kidney disease. Kidney Int 2014;86:896-904.

52. Kohan DE, Pritchett $Y$, Molitch M, Wen S, Garimella T, Audhya P, et al. Addition of atrasentan to renin-angiotensin system blockade reduces albuminuria in diabetic nephropathy. J Am Soc Nephrol JASN 2011;22:763-72.

53. Heerspink HJL, Parving HH, Andress DL, Bakris G, Correa-Rotter $\mathrm{R}$, Hou FF, et al. Atrasentan and renal events in patients with type 2 diabetes and chronic kidney disease (SONAR): a doubleblind, randomised, placebo-controlled trial. Lancet 2019;393: 1937-47. 